\title{
Economic concerns about global healthcare in lung, head and neck cancer: meeting the economic challenge of predictive, preventive and personalized medicine
}

\author{
Christine Ausweger • Eva Burgschwaiger • Andrea Kugler • Reinhard Schmidbauer • \\ Isabell Steinek • Yordan Todorov • Dietmar Thurnher $\cdot$ Kurt Krapfenbauer
}

Received: 13 September 2010 / Accepted: 1 November 2010 /Published online: 3 December 2010

(C) European Association for Predictive, Preventive and Personalised Medicine 2010

\begin{abstract}
The introduction of biological science into the practice of medicine was a big transforming event for the profession, leading to different new medical models such as predictive, preventive and personalized medicine. Each of them is a rapidly emerging field that helps us to determine the risk for individuals to develop specific diseases, detect the disease's earliest onset and prevent or intervene early enough to provide maximum benefit for each patient. However, to realize this new potential, new healthcare models must be created, improved and validated. New healthcare models that are more proactive than reactive because prevention is less expensive than reactive medicine. Current knowledge about predictive, preventive and personalized medicine is already sufficient to implement this approach, but there are no effective practice models, delivery systems and appropriate reimbursement mechanisms. In the course of this review, we describe the economic components and benefits of a predictive, preventive and personalized health plan for lung as well as head and neck cancer and show how prospective care could relate to a community or group of covered individuals.
\end{abstract}

D. Thurnher is National Representative of EPMA in Austria.

C. Ausweger · E. Burgschwaiger - A. Kugler ' R. Schmidbauer •

I. Steinek $\cdot$ Y. Todorov

University of Economics and Business,

Althanstrasse 14,

Vienna A-1090, Austria

D. Thurnher $\cdot$ K. Krapfenbauer $(\bowtie)$

Department of Otorhinolaryngology, Head and Neck Surgery,

Medical University of Vienna,

Waehringer Guertel 18-20,

Vienna A-1090, Austria

e-mail: kurt.krapfenbauer@boehringer-ingelheim.com
Keywords Economic challenge $\cdot$ In vitro diagnostics $\cdot$ Lung cancer·Head and neck cancer. Prospective medicine

\section{Introduction}

Head and neck carcinoma (HNC) is the sixth most common malignancy and a major cause of cancer morbidity and mortality worldwide. Globally about 500,000 new oral and pharyngeal cancers are diagnosed annually and three quarters of these are from the developing world [1]. Age standardized incidence rate of $\mathrm{HNC}$ in males exceeds 30 in 100,000 populations in regions of France, Hong Kong, the Indian subcontinent, Central/Eastern/Southern Europe, regions of South America and among USA blacks. High rates (higher than 10 in 100,000 populations) in females are found in India and Hong Kong. Due to sedentary lifestyle (e.g. heavily smoking), unhealthy nutrition (abnormal consumption of spices, strong alcohols, etc.) and a prolonged lifespan, the prevalence of the disease in the developed and developing countries is further increasing [2]. One main reason for the high prevalence is that $\mathrm{HNC}$ is normally a late-diagnosed disease and is usually only discovered when the first complications occur. Therapy of advanced $\mathrm{HNC}$ in the late state is far away from therapeutic success. Five-year survival rates for mouth, tongue, oropharynx and laryngopharyngeal cancers seldom exceed $40 \%$. Quick diagnostic measures and/or early prevention are therefore critical for the control of the disease status. The market size and huge demand for these tests make it a model disease to develop new approaches to in-vitro diagnostic (IVD). In this review, we briefly summarize the overview of IVD for lung cancer and $\mathrm{HNC}$, the current commercial devices available for, and the recent work in 
the area of artificial receptors and the potential for the development of new devices for cancer diagnosis.

Current status of lung as well as head and neck cancer

Every year more than one million new cases of HNC are diagnosed worldwide and approximately half of those people die. However, 1/3 of them could be saved if the disease had been diagnosed at an earlier stage. Current used radiological or clinical methods like MRI, CT, or even PETCT or clinical methods like bronchoscopy are expensive, might be discomforting and are more often than not inaccurate to diagnose $\mathrm{HNC}$ and lung cancer at an early stage. The costs of treatment for a lung cancer and $\mathrm{HNC}$ patient are between $€ 17,000$ and $€ 22,000$ [3-7]. Through early detection, those costs can be significantly reduced. Thus, there is an acute need for an early, accurate, painless and cost-saving diagnostic method.

\section{Customer need}

The traditional process of diagnosing a disease is a complicated one and involves up to four different parties, depending on the condition [1] (Fig. 1).

A patient will consult his physician with certain symptoms (e.g. pain, bleeding, coughing or dysphagia) already present. Based on these symptoms a physician will make a hypothesis what the disease may be. However, to prove his theory he often requires specialized laboratory tests. So, after a clinical exam samples will be taken and examined by any diagnostic laboratory. When the test results are available a treatment will be assigned by the physician. This treatment may prove to be accurate and leads to a stabilization/healing process or may be inaccurate and will require further investigation. On the monetary side, as far as the patient is insured, the costs for diagnosis are born by the health insurer; i.e. patients have to pay indirectly for diagnostic tests through their health insurance contributions. However, the health insurer is only willing to pay if laboratories are using state-of-the-art diagnostic methods (personal communication: Prof. D. Thurnher, Medical University of Vienna, April 2010). There are several problems arising from this traditional method of diagnosing for the parties involved, which will be considered as paying and receiving customers.

Paying customers

\section{Diagnostic laboratories}

Current diagnostic methods are time consuming. They normally require hours until the result can be validated. Furthermore, high costs can be associated with diagnostic instruments like CT (especially purchasing costs) (personal communication: Prof. D. Thurnher, Medical University of Vienna, April 2010). Therefore, it is clear that a faster and cost-efficient instrument is needed. This would cut processing times and associated costs.

Current diagnostics instruments are not always able to deliver an accurate diagnosis to the doctor (e.g. cancer must be large enough in order to be seen with CT) (personal communication: Prof. D. Thurnher, Medical University of Vienna, April 2010) and may lead to a wrong treatment. So, an accurate diagnostic instrument is also needed in those institutions.

\section{Public and private health insurers}

The late application of current diagnostic methods after symptoms already have occurred can imply higher treatment costs (longer hospital stays, etc.) especially if diseases are diagnosed in a late stage [3]. It has been estimated for western European countries that one lung cancer patient costs approximately $€ 17,000$ and one $\mathrm{HNC}$ patient approximately $€ 22,000$ [4-7]. As a result, earlier and accurate treatment would significantly shorten those costs (personal communication according to expert interview, Wiener Gebietskrankenkasse,
Fig. 1 Traditional process of diagnosis

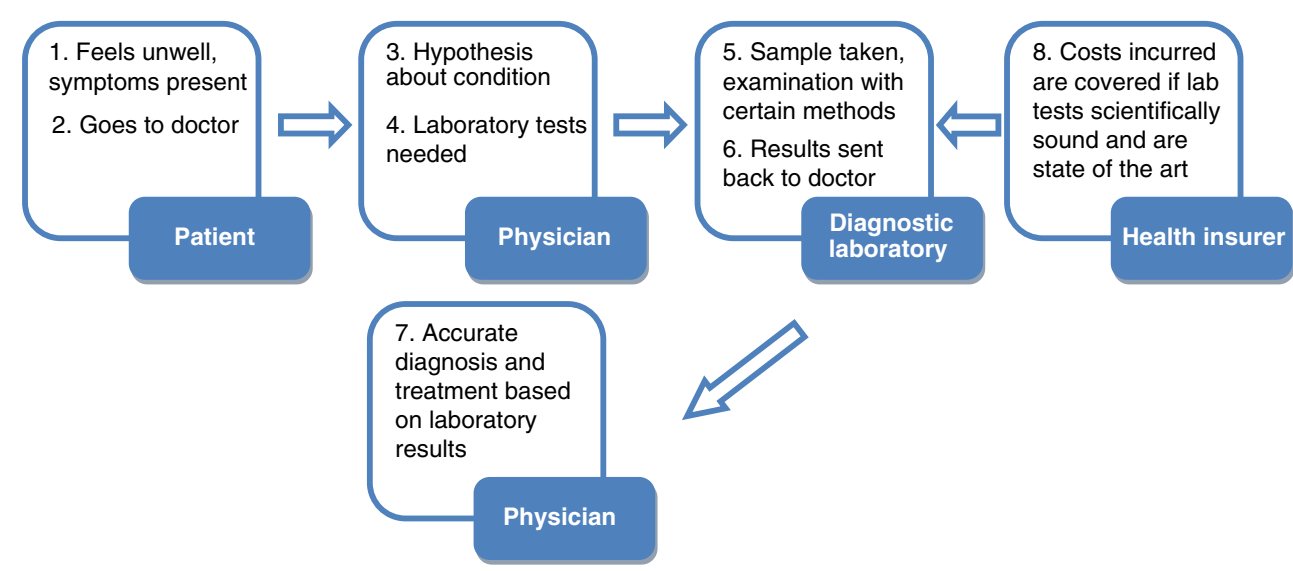


April 2010). Many public health insurers are currently experiencing financial deficits and cannot sustain their operations in the long run without cash injections. Thus, such an early diagnostic instrument may solve this problem (personal communication; Prof. D. Thurnher, Medical University of Vienna, April 2010).

\section{Receiving customers}

Current diagnostic methods are used when symptoms are already present. Therefore, diseases are often diagnosed in a late stage, which means a lower survival chance for patients [8]. The sooner a (potential) disease is diagnosed-even before the symptoms occur - the faster and more accurate the treatment can be. This in turn contributes to a prolonged life expectancy of the patient [9].

Traditional forms of diagnostic instruments may be inaccurate. An inaccurate treatment assigned because of a wrong diagnosis can be very harmful to the patient. Therefore, using early diagnostic instruments, an inaccurate treatment can be prevented before it causes serious damage [10].

The need for a more accurate, cost-saving and fast diagnostic method is acute. Especially when we look at our market, cancer mainly caused by smoking, the following customer pain and need can further be identified.

Cancer caused by smoking is usually found in the upper aero-digestive tract (head and neck) or in the lungs. It is usually diagnosed through X-Ray, CT and endoscopy (e.g. bronchoscopy), as soon as symptoms have occurred (e.g. coughing up phlegm with traces of blood (personal communication: Prof. D. Thurnher, Medical University of Vienna, April 2010)). The problems with the traditional diagnostic methods are:

- Inaccuracy, as cancer in early stages is not visible with CT and X-Ray;

- Discomfort and potential morbidity for patients when using invasive diagnostic procedures;
- Costs incurred when using an expensive diagnostic instrument like the CT.

\section{Market overview}

In vitro diagnostics market (IVD)

IVD market plays a major role in healthcare and disease management. It affects around $70 \%$ of healthcare decisions. The global IVD market is estimated to generate sales of nearly $\$ 43.2 \mathrm{bn}$ in 2010 and $\$ 54 \mathrm{bn}$ by 2013 . The global market is expected to grow with a CAGR (Compound Annual Growth Rate) of 7.1\% and the European with 7\%. Consequently, the European IVD market is expected to reach ca. \$ 16bn in 2010 and \$ 19.6bn in 2013. On the whole, the European IVD market represents approximately $37 \%$ of the global IVD market in 2010 [11] (Fig. 2).

The global cancer IVD market has reached $\$ 7,4 \mathrm{bn}$ in 2009 [12] and is expected to reach $\$ 7.9 \mathrm{bn}$ in 2010. Under the assumption that $37 \%$ of the global cancer IVD market represents the European cancer IVD market and a CAGR of $7 \%$, the latter is expected to reach $\$ 2.9 \mathrm{bn}$ in $2010, \$ 3.8 \mathrm{bn}$ in 2014 and \$ 4.7bn in 2017.

\section{Cancer market}

In 2008 the global cancer market was valued at $\$ 47.7 \mathrm{bn}$ and is expected to reach $\$ 76.7 \mathrm{bn}$ in 2014 . This represents a CAGR of $8.2 \%$ between 2008 and 2014 [13]. Cancer as a leading cause of death is projected to continue rising with an estimated $12 \mathrm{~m}$ deaths in 2030 [14]. The World Health Organization (WHO) notes that one third of all cancer diseases could be prevented by avoiding all cancer-related risk factors. Another third could be cured, if only diagnosed early and treated efficiently [15]. However, only $30-50 \%$ of all cancer patients suffer from pain at the time of diagnosis

Fig. 2 Global IVD market

Global IVD Market 2008 - 2013 (E)

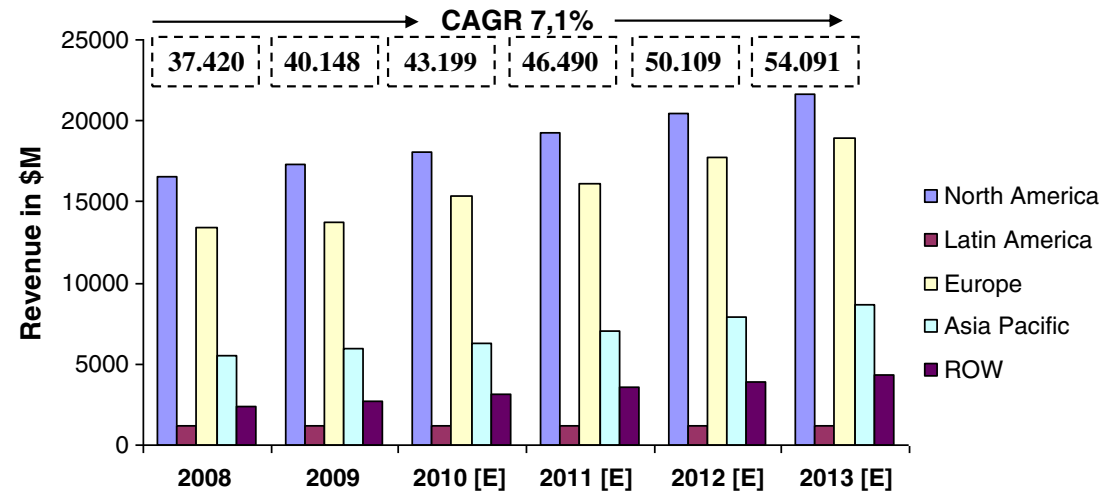


[16]. Therefore, the goal of the predictive medicine is the early pre-symptomatic detection of diseases.

Concerning the European cancer market, 3.2 million Europeans are diagnosed with cancer each year. It is the second most common cause of death in Europe [17].

\section{Lung cancer and $\mathrm{HNC}$}

There are about one million incidences of lung cancer each year worldwide. $90 \%$ of them lead to death and $85 \%$ of those are caused by smoking [9]. The annual incidence rate for lung cancer in Europe accounts for 60 per 100,000 inhabitants (approximately 310,000 new cases) [18].

In Europe approximately 100,800 new HNC cases are diagnosed annually. Around 40,000 of them die, which makes up $40 \%$ [13]. However, the incidence rate of HNC has doubled within the past 20 years due to the rising tobacco and alcohol consumption and is therefore further expected to rise [3]. About $85 \%$ of this cancer can be related to tobacco. Furthermore, excessive alcohol consumption is another risk factor for developing HNC [3]. However, significant advances and improvements can be observed in tackling cancer within Europe. Nevertheless, cancer remains a serious public health concern, which is also putting a heavy financial burden on the society.

\section{Financial burden of lung cancer and $\mathrm{HNC}$}

In 2001 it has been estimated that the treatment of a patient with lung cancer costs $€ 17,000$. These costs are split into hospitalization which accounts for almost $50 \%$ and $13 \%$ of chemotherapy [3]. Furthermore, the Institute for Medical Technology Assessment at the University in Rotterdam calculated the costs of HNC. Diagnosis, treatment and 2 years of follow-up of patients with a primary tumor were calculated with $€ 21,858$. For patients with a recurrent tumor, the costs were $€ 27,629$. The 10 years follow-up costs were $€ 423$. In total, the average costs of a new patient were $€ 31,829$ [6]. Through early detection of this diseases and early treatment such costs can be reduced significantly.

\section{Conclusion}

In addition to their original healing-mission, physicians gain more and more responsibility in health politics including most important aspects like education and medical ethics. Recent developments and possibilities such as in prenatal diagnostics, molecular biology and in vitrofertilization demand clear cut positions from the medical staff. Political evaluation of costs in healthcare systems concerns analysis of the most common factors responsible for the increasing costs. High expectations and demands of the patients are one reason, other are excessive use of new technologies, increase of the aged in the population and growing cost for liability cases. Mechanisms for the downregulation of costs are restricted. The so-called free market in healthcare such as premium-competition of health insurance point out more theoretical than practical aspects. The Health insurance law (KVG) by increasing the extent of services has contributed to a further increase of costs instead of lowering them. Shifting from hospitalization to out-patient treatment and reducing beds for acute services could lower hospital costs further. AIDS, Drug-politics and environmental destruction, three actual plagues, represent further political challenges for physicians. For the future a reasonable politic involving a better motivated medical corps has to be promoted. To this end first personal structures should be strengthened by help of new ethical view points. Future medicine should be more proactive rather than reactive. Therefore medical responsibility in political development is mandatory.

\section{Outlook}

There is a long continuum of differing positions amongst academia, the private sector and the public sector in determining the appropriate balance between providing incentives to both innovator drug companies and generic drug manufacturers, while at the same time also protecting legitimate patent rights. Juxtaposed to these issues is the fact that the cost of biomedical research is enormous and it continues to grow as the government seeks to provide for the health and welfare of a public that not only seeks therapeutic medicine and medical care when sick, but also wants prophylactic and diagnostic products. Furthermore, personalized medicine, resulting from the advances in functional genomics and new and increased use of lifestyle drugs providing therapies is indicative of the ever-increasing expectations for a greater range of products. The debate then is not only how to make such products available but also how to make them affordable. With more than 500 drug patents scheduled to expire between 2004 and 2009 (with 26 of these drugs accounting for more than 38 billion dollars in sales annually), the stakes are high, for not only the generic and brand name manufacturers, but also for the consumer.

\section{References}

1. Gupta PC, Pindborg JJ, Mehta FS. Comparison of carcinogenicity of betel quid with and without tobacco: an epidemiological review. Ecol Dis. 1982;1:213-9.

2. Fearlay J, Bray F, Pisani P, Parkin DM. Cancer incidence, mortality and prevalence worldwide. Version 1.0. IARC Cancer Base No.5., IARC Press, Lyon; 2001. 
3. European Lung Foundation, http://www.european-lung-foundation. org/index.php?id=65.

4. van Agthoven M, van Ineveld BM, de Boer MF, Leemans CR, Knegt PP, Snow GB, et al. The costs of head and neck oncology: primary tumors, recurrent tumors and long-term follow-up. Eur J Cancer. 2001;37(17):2204-11.

5. European diagnostics manufacturers association, http://www. edma-ivd.be/index.php?id=467.

6. Cancer Research, http://www.cancerhelp.org.uk/type/lung-cancer/ about/lung-cancer-symptoms.

7. Immeasure, http://www.immeasure.com/Investor.htm.

8. Drugresearcher, http://www.drugresearcher.com/Researchmanagement/Cancer-diagnostics-to-reach-7.4-Billion-by-2009.

9. Business Insights (2009), The Cancer Market Outlook to 2014.

10. WHO, http://www.who.int/cancer/en/.
11. AKH consilium, http://akh-consilium.at/indikation/Malignome.

12. Österreichische Krebshilfe Wien, http://www.krebshilfe-wien.at/ Frueherkennung.99.0.html.

13. Health-EU, http://ec.europa.eu/health/major_chronic_diseases/ diseases/cancer/index en.htm.

14. OeGHO, http://www.krebsfacharzt.at/ueber-krebs/lungenkrebs/ lungenkrebs-im-ueberblick.html.

15. AKH consilium, http://akh-consilium.at/indikation/Bronchialkarzinom/ Diagnose.

16. Mouth Cancer Foundation, http://www.rdoc.org.uk/.

17. Ferlay J, Parkin DM, Steliarova-Foucher E. Estimates of cancer incidence and mortality in Europe in 2008. Eur J Cancer. 2010;46 (4):765-81.

18. The University of York (2004), http://www.york.ac.uk/inst/crd/ EHC/ehc85.pdf. 